\title{
The Influence of Snare Size on the Utility and Safety of Cold Snare Polypectomy for the Removal of Colonic Polyps in Japanese Patients
}

\author{
Hisatsugu Noda ${ }^{\mathrm{a}}$, Naotaka Ogasawara ${ }^{\mathrm{a}, \mathrm{b}}$, Tomoya Sugiyama ${ }^{\mathrm{a}}$, Takashi Yoshimine ${ }^{\mathrm{a}}$, \\ Yasuhiro Tamura ${ }^{\text {a }}$, Shinya Izawa ${ }^{\text {a }}$, Yoshihiro Kondo ${ }^{\text {a }}$, Masahide Ebi ${ }^{\text {a }}$, Yasushi Funaki ${ }^{a}$,

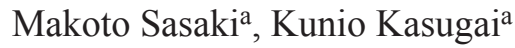

\begin{abstract}
Background: Cold snare polypectomy (CSP) has been recently reported to be useful for the removal of small colonic polyps. However, the relationship between the histologically complete resection rate and snare size used during CSP has not been reported. Our aim was to assess the utility of CSP.
\end{abstract}

Methods: We analyzed the histologically complete resection rates and the frequency of complications for 175 colon polyps removed by CSP. Moreover, we examined the histologically complete resection rate associated with different snare sizes used during CSP.

Results: There was no significant difference in the histologically complete resection rate between endoscopic mucosal resection (EMR) (60.9\%) and CSP (53.1\%). There were also no significant differences in the frequency of complications including perforation and postoperative bleeding between EMR (perforation: none; postoperative bleeding: two patients) and CSP (perforation: none; postoperative bleeding: none). Histological examination revealed that the complete resection rate of CSP using a short snare (61.6\%) was significantly higher than that of CSP using a long snare $(44.9 \% ; \mathrm{P}<0.05)$. There were no significant differences in the frequency of complications between CSP using the short snare and that using the long snare.

Conclusions: CSP is a safe, useful method for the removal of colonic polyps. CSP using the short snare improved the histologically complete resection rates compared to the long snare. Future studies to further assess the utility of CSP are required.

Keywords: Cold snare polypectomy; Colon polyp; Colon cancer; Endoscopic submucosal resection; Snare size

\footnotetext{
Manuscript accepted for publication July 06, 2016

aDepartment of Gastroenterology, Aichi Medical University School of Medicine, 1-1 Yazakokarimata, Nagakute, Aichi, Japan

${ }^{b}$ Corresponding Author: Naotaka Ogasawara, Department of Gastroenterology, Aichi Medical University School of Medicine, 1-1 Yazakokarimata, Nagakute, Aichi 480-1195, Japan. Email: nogasa@aichi-med-u.ac.jp
}

doi: http://dx.doi.org/10.14740/jocmr2646w

\section{Introduction}

Colorectal cancer is the second leading cause of cancer-related death in the world [1-3]. Colonoscopy has become a primary screening test for colorectal cancer, and polypectomy of colon polyps is considered an effective treatment, reducing the risk of colon cancer development by interrupting the progression of adenoma to adenocarcinoma [1-3]. Colonoscopic polypectomy with endoscopic mucosal resection (EMR) is a very effective technique for the prevention of colorectal cancer $[4,5]$. Polypectomy with EMR is considered safe, but is associated with a low risk of polypectomy-related issues such as bleeding and perforation [6,7]. Recently, cold snare polypectomy (CSP) has been reported to be useful for the removal of small colon polyps $[8,9]$. CSP requires a shorter procedure time and eliminates concerns associated with electrocautery-related tissue damage and post-polypectomy cautery syndrome [10]. The cold snare technique has been recommended for the removal of small polyps owing to its safety profile, speed of resection, and effectiveness $[8$, 9]. In Japan, EMR is the most common method for removing small colonic polyps; however, CSP has gradually been introduced. Only few reports regarding the utility and safety of CSP in Japanese patients exist [10-13]. Horiuchi et al reported that delayed bleeding requiring hemostasis after colon polypectomy occurred significantly less often with CSP than with EMR [11]. Two studies comparing the types of snares used during CSP have been published [13, 14]. One report demonstrated that CSP with a thin wire snare was more effective than that with a thick-wired snare, although differences in the completeness of the histological excision were not statistically significant [14]. Another report demonstrated that a CSP snare with a thinner wire and/or of a shield-like shape was more effective than the traditional CSP snare [13]. However, there has been no report on the relationship between the histologically complete resection rate and the snare size used during CSP.

In the present study, we retrospectively evaluated the histologically complete resection rates of colon polyps removed by CSP and the complications associated with CSP compared with EMR. Moreover, we examined the histologically complete resection rate and frequency of complications in terms of 
Table 1. Comparison of Characteristics of Patients in the EMR and CSP Groups

\begin{tabular}{llll}
\hline & EMR & CSP & P value \\
\hline Number of patients & 423 & 106 & \\
Sex (male/female) & $285 / 138$ & $83 / 23$ & $<0.05$ \\
Mean age in years (range) & $67.9(35-90)$ & $66.8(40-85)$ & $\mathrm{NS}$ \\
Hypertension (presence/absence) & $169 / 254$ & $37 / 69$ & $\mathrm{NS}$ \\
Diabetes mellitus (presence/absence) & $76 / 347$ & $23 / 83$ & $\mathrm{NS}$ \\
Liver disease (presence/absence) & $17 / 406$ & $6 / 100$ & $\mathrm{NS}$ \\
Hemodialysis (presence/absence) & $12 / 411$ & $4 / 102$ & $\mathrm{NS}$ \\
Other comorbidities (presence/absence) & $97 / 326$ & $18 / 88$ & $\mathrm{NS}$ \\
Usage of an anticoagulant and/ & 22 & 19 & $\mathrm{NS}$ \\
or antiplatelet drugs (\%) & & & \\
\hline
\end{tabular}

EMR: endoscopic mucosal resection; CSP: cold snare polypectomy; NS: not significant.

Table 2. Comparison of Polyp Characteristics Between the EMR and CSP Groups

\begin{tabular}{llll}
\hline & EMR & CSP & P value \\
\hline Number of lesions & 1,010 & 175 & \\
Mean lesion size in mm (range) & $6.8(3-15)$ & $5.0(3-10)$ & NS \\
Macroscopic type of polyp (sessile/semipedunculated/pedunculated) & $582 / 323 / 105$ & $135 / 40 / 0$ & $<0.05$ \\
Histological type of polyp (adenocarcinoma/adenoma/hyperplasia) & $18 / 905 / 87$ & $0 / 157 / 18$ & NS \\
\hline
\end{tabular}

EMR: endoscopic mucosal resection; CSP: cold snare polypectomy; NS: not significant.

the snare size used during CSP.

\section{Methods}

\section{Patients}

A total of 423 consecutive patients (285 male and 138 female; mean age, 67.9 years) with 1,010 lesions (range of polyp size, 3 - $15 \mathrm{~mm}$; average polyp size, $6.8 \mathrm{~mm}$; macroscopic type, 582 lesions of sessile type, 323 lesions of semipedunculated type, and 105 lesions of pedunculated type) resected by EMR and 106 consecutive patients (83 male and 23 female; mean age, 66.8 years) with 175 lesions (range of polyp size, 3 - $10 \mathrm{~mm}$; average polyp size, $5.0 \mathrm{~mm}$; macroscopic type, 135 lesions of sessile type and 40 lesions of semipedunculated type) resected by CSP at Aichi Medical University School of Medicine between May 2014 and June 2015 were recruited. Moreover, the lesions resected by CSP were divided into two groups: 89 using a long snare (diameter, $27 \mathrm{~mm}$ ) between May 2014 and November 2014 and 86 using a short snare (diameter, $13 \mathrm{~mm}$ ) between December 2014 and June 2015.

The Ethics Committee of Aichi Medical University School of Medicine granted approval for this study, and written informed consent for participation in all the study procedures was obtained from all patients.

\section{EMR and CSP procedures}

EMR and CSP procedures were performed according to our institutional methods. EMR was principally indicated in polyps of $20 \mathrm{~mm}$ or less in diameter and CSP was indicated in polyps of $3-10 \mathrm{~mm}$ in diameter. EMR and CSP were performed using either a long snare (Captivator $27 \mathrm{~mm}$, Boston Scientific, Tokyo, Japan) or a short snare (Captivator $13 \mathrm{~mm}$, Boston Scientific, Tokyo, Japan).

Table 3. Comparison of the Histologically Complete Resection Rate and Frequency of Complications in the EMR and CSP Groups

\begin{tabular}{llll}
\hline & EMR & CSP & P value \\
\hline Histologically complete resection rate (\%) & 60.9 & 53.1 & NS \\
Postoperative bleeding (\%) & 0.2 & 0 & NS \\
Perforation (\%) & 0 & 0 & NS \\
\hline
\end{tabular}

EMR: endoscopic mucosal resection; CSP: cold snare polypectomy; NS: not significant. 
Table 4. Comparison of Polyp Characteristics Based on Snare Length

\begin{tabular}{llll}
\hline & CSP using short snare & CSP using long snare & P value \\
\hline Number of lesions & 86 & 89 & NS \\
Macroscopic type of polyp (sessile/semipedunculated) & $68 / 18$ & $67 / 22$ & NS \\
Histological type of polyp (adenoma/hyperplasia) & $80 / 6$ & $77 / 12$ & \\
\hline
\end{tabular}

CSP: cold snare polypectomy; NS: not significant.

\section{Evaluation of complete resection rate}

The EMR and CSP procedures were defined as a histologically complete resection when a horizontal margin and a vertical margin of the resected polyp were histologically negative. The EMR and CSP procedures were defined as a histologically incomplete resection when a horizontal margin and a vertical margin of resected polyp were histologically positive or uncertain.

\section{Statistical analysis}

The results are presented as the mean \pm SD for quantitative data and as percentages for categorical data. Quantitative data were analyzed using the Mann-Whitney U test and categorical data were analyzed using the $\chi^{2}$ test or Fisher's exact test. All statistical analyses were performed in JMP version 9.02 for Windows software (SAS Institute Inc., Cary, NC, USA). A Pvalue $<0.05$ was considered significant.

\section{Results}

\section{Comparison between EMR and CSP}

Four hundred and twenty-three patients underwent EMR and 106 patients underwent CSP. There were significant differences in sex between the two groups (Table 1). However, there were no significant differences in other background factors (Table 1). One thousand and ten polyps were resected by EMR and 175 polyps were resected by CSP (Table 2). There were no significant differences in the mean size or histological type of the colon polyps between EMR and CSP groups (Table $2)$. However, the frequency of pedunculated or semipedunculated type polyps in the EMR group was significantly higher than that in the CSP group $(\mathrm{P}<0.05)$ (Table 2$)$. There was no significant difference in the histologically complete resection rate between EMR and CSP (Table 3). There was also no sig- nificant difference in the frequency of complications including postoperative bleeding and perforation between EMR and CSP (Table 3).

\section{Comparison between short snare and long snare}

Eighty-six polyps were resected by CSP using the short snare and 89 polyps were resected by CSP using the long snare (Table 4). There were no significant differences in the macroscopic type or histological type of the polyps between the two groups (Table 4). However, the histologically complete resection rate of CSP with the short snare was significantly higher than that of CSP with the long snare $(\mathrm{P}<0.05)$ (Table 5). In contrast, there was no significant difference in the frequency of complications between CSP with the short snare and with the long snare (Table 5).

\section{Discussion}

CSP was first reported to be a safe excision method for small polyps in 1992 [2]. In 2005, the efficacy of CSP for the removal of small colon polyps was reported with the conclusion that cold snare removal of colon polyps was associated with a high retrieval rate [15]. A prospective multicenter study also reported that postoperative bleeding did not occur in CSP of 1,015 colon polyps [16]. Chang et al reported that CSP was superior to cold forceps polypectomy with regard to the completeness of polypectomy and was a less time-consuming procedure [17]. Therefore, the cold snare technique has been recommended for the removal of small polyps owing to its safety profile, speed of resection, and effectiveness $[8,9]$.

In Japan, additional studies demonstrated that CSP requires a shorter procedure time and eliminates concerns associated with electrocautery-related tissue damage and post-polypectomy cautery syndrome [10]. Moreover, a randomized controlled trial from a single institution found that postoperative bleeding occurred in $14 \%$ of patients receiving hot polypectomy with EMR, whereas it did not occur in the CSP group [11]. An-

Table 5. Comparison of the Histologically Complete Resection Rate and Frequency of Complications by CSP Snare Length

\begin{tabular}{llll} 
& CSP using short snare & CSP using long snare & P value \\
\hline Histologically complete resection rate (\%) & 61.6 & 44.9 & $<0.05$ \\
Complications & 0 & 0 & NA \\
\hline
\end{tabular}

CSP: cold snare polypectomy; NA: not applicable. 
other report confirmed that the post-bleeding risk of CSP was extremely low compared with that of hot polypectomy with EMR. These previous reports concluded that neither difficulty in polyp retrieval nor bleeding after CSP was a problem encountered with CSP $[10,11]$. Ichise et al demonstrated that the frequency of abdominal symptoms after hot polypectomy with EMR was higher than that after CSP even when no perforation or bleeding requiring endoscopic hemostasis occurred [10]. In our study, no perforation occurred in either the EMR or the CSP group. In contrast, two patients had postoperative bleeding $(0.2 \%)$ in the EMR group, whereas none in the CSP group had. However, this difference was not statistically significant. Therefore, CSP can be considered, at least, as safe as EMR, similar to the conclusions from a previous report [11].

Several studies have evaluated the histologically complete resection rate of colon polyps removed by CSP with rates ranging widely from $44.1 \%$ to $91 \%[13,14]$. The wide range of the histologic complete resection rate in CSP may depend on the technical skill involved, the treatment process for the specimens of the resected colon polyps, or the diagnosing abilities among pathologists. However, the data on the histologic complete resection rate in CSP are limited, and further studies are needed to better understand the factors influencing the rate and to elucidate the accurate rate of histologically complete resection in CSP. In the present study, the histologically complete resection rates were $53.1 \%$ in CSP and $60.9 \%$ in EMR. The histologically complete resection rate of CSP in our results was similar to that in previous reports. However, to our knowledge, no study has reported the histologically complete resection rate of colon polyps resected by EMR; thus, we could not compare our EMR rate with that of previous reports.

Given the lack of reports on the influence of the size of the polypectomy snare used during CSP on the histologically complete resection rate, we compared the obtained by CSP with a long snare and by CSP with a short snare. The results demonstrated that the histologically complete resection rate using the short snare was significantly higher than that using the long snare. Therefore, CSP with the short snare is more useful compared with CSP using the long snare. Smaller snares, such as that used in our study, appears to be more appropriate and is recommended for use for the removal of colon polyps less than $10 \mathrm{~mm}$. The higher complete resection rate in CSP using the short snare may be due to the ability to impose the short snare upon the normal colon mucosae surrounding the polyps more strongly compared with the long snare, thus securing more normal mucosae surrounding the polyps as safety margins and avoiding the leaving behind of polyps, especially at the boundary lesion between the normal mucosa and the polyp.

Limitations of the present study include the use of retrospective data from a single center and the relatively small number of polyps. However, this study is the first report to compare the histologically complete resection rates between EMR and CSP. Moreover, our study demonstrated that snare size influenced the histologically complete resection rates in CSP.

In conclusion, CSP is a useful, safe method for the removal of colonic polyps without complications, with performance equivalent to that of EMR. The histologically complete resection rate with CPS using the short snare is significantly higher than that with CPS using the long snare. Thus, our study demonstrates that the short snare is more useful for the removal of colonic polyps compared to the long snare. More studies are needed to further elucidate the utility of CSP and also to develop more appropriate devices and methods for CSP.

\section{Conflicts of Interest}

Kunio Kasugai received research grant from AstraZeneca K.K., Daiich Sankyo Co., Ltd, Takeda Pharmaceutical Co., Ltd and lecture fees from AstraZeneca K.K., Daiich Sankyo Co., Ltd, Takeda Pharmaceutical Co., Ltd.

\section{References}

1. Robertson DJ. Colonoscopy for colorectal cancer prevention: is it fulfilling the promise? Gastrointest Endosc. 2010;71(1):118-120.

2. Tappero G, Gaia E, De Giuli P, Martini S, Gubetta L, Emanuelli G. Cold snare excision of small colorectal polyps. Gastrointest Endosc. 1992;38(3):310-313.

3. Zauber AG, Winawer SJ, O'Brien MJ, Lansdorp-Vogelaar I, van Ballegooijen M, Hankey BF, Shi W, et al. Colonoscopic polypectomy and long-term prevention of colorectal-cancer deaths. N Engl J Med. 2012;366(8):687-696.

4. Winawer SJ, Zauber AG, Ho MN, O'Brien MJ, Gottlieb LS, Sternberg SS, Waye JD, et al. Prevention of colorectal cancer by colonoscopic polypectomy. The National Polyp Study Workgroup. N Engl J Med. 1993;329(27):19771981.

5. Citarda F, Tomaselli G, Capocaccia R, Barcherini S, Crespi M. Efficacy in standard clinical practice of colonoscopic polypectomy in reducing colorectal cancer incidence. Gut. 2001;48(6):812-815.

6. Gatto NM, Frucht H, Sundararajan V, Jacobson JS, Grann VR, Neugut AI. Risk of perforation after colonoscopy and sigmoidoscopy: a population-based study. J Natl Cancer Inst. 2003;95(3):230-236.

7. Anderson ML, Pasha TM, Leighton JA. Endoscopic perforation of the colon: lessons from a 10-year study. Am J Gastroenterol. 2000;95(12):3418-3422.

8. Hewett DG, Rex DK. Colonoscopy and diminutive polyps: hot or cold biopsy or snare? Do I send to pathology? Clin Gastroenterol Hepatol. 2011;9(2):102-105.

9. Hewett DG. Colonoscopic polypectomy: current techniques and controversies. Gastroenterol Clin North Am. 2013;42(3):443-458.

10. Ichise Y, Horiuchi A, Nakayama Y, Tanaka N. Prospective randomized comparison of cold snare polypectomy and conventional polypectomy for small colorectal polyps. Digestion. 2011;84(1):78-81.

11. Horiuchi A, Nakayama Y, Kajiyama M, Tanaka N, Sano K, Graham DY. Removal of small colorectal polyps in anticoagulated patients: a prospective randomized comparison of cold snare and conventional polypectomy. Gastrointest Endosc. 2014;79(3):417-423.

12. Uraoka T, Ramberan H, Matsuda T, Fujii T, Yahagi N. 
Cold polypectomy techniques for diminutive polyps in the colorectum. Dig Endosc. 2014;26(Suppl 2):98-103.

13. Horiuchi A, Hosoi K, Kajiyama M, Tanaka N, Sano K, Graham DY. Prospective, randomized comparison of 2 methods of cold snare polypectomy for small colorectal polyps. Gastrointest Endosc. 2015;82(4):686-692.

14. Din S, Ball AJ, Riley SA, Kitsanta P, Johal S. Cold snare polypectomy: does snare type influence outcomes? Dig Endosc. 2015;27(5):603-608.

15. Deenadayalu VP, Rex DK. Colon polyp retrieval after cold snaring. Gastrointest Endosc. 2005;62(2):253-256.

16. Repici A, Hassan C, Vitetta E, Ferrara E, Manes G, Gullotti G, Princiotta A, et al. Safety of cold polypectomy for $<10 \mathrm{~mm}$ polyps at colonoscopy: a prospective multicenter study. Endoscopy. 2012;44(1):27-31.

17. Lee CK, Shim JJ, Jang JY. Cold snare polypectomy vs. Cold forceps polypectomy using double-biopsy technique for removal of diminutive colorectal polyps: a prospective randomized study. Am J Gastroenterol. 2013;108(10):1593-1600. 